\title{
Trajectory of body shape in early and middle life and all cause and cause specific mortality: results from two prospective US cohort studies
}

\author{
Mingyang Song, ${ }^{1,2}$ Frank B Hu, 2,3 Kana Wu, ${ }^{2}$ Aviva Must, ${ }^{4}$ Andrew T Chan, ${ }^{1,3}$ Walter C Willett, $, 2,3$ \\ Edward L Giovannucci²,3
}

${ }^{1}$ Clinical and Translational

Epidemiology Unit and Division

of Gastroenterology,

Massachusetts General Hospital

and Harvard Medical School,

Boston, MA02114, USA

${ }^{2}$ Departments of Nutrition and

Epidemiology, Harvard T H Chan

School of Public Health, Boston,

MA 02115, USA

${ }^{3}$ Channing Division of Network

Medicine, Department of

Medicine, Harvard Medical

School, Boston, MA 02115, USA

${ }^{4}$ Department of Public Health

and Community Medicine, Tufts

University School of Medicine,

Boston, MA 02111, USA

Correspondence to: M Song

msong2@mgh.harvard.edu

Additional material is published

online only. To view please visit

the journal online.

Cite this as: BMJ 2016;353:i2195 http://dx.doi.org/10.1136/bmj.i2195

Accepted: 7 April 2016

\section{ABSTRACT}

\section{OBJECTIVE}

To assess body shape trajectories in early and middle life in relation to risk of mortality.

DESIGN

Prospective cohort study.

SETTING

Nurses' Health Study and Health Professionals

Follow-up Study.

\section{POPULATION}

80266 women and 36622 men who recalled their body shape at ages $5,10,20,30$, and 40 years and provided body mass index at age 50 , followed from age 60 over a median of 15-16 years for death.

\section{MAIN OUTCOME MEASURES}

All cause and cause specific mortality.

RESULTS

Using a group based modeling approach, five distinct trajectories of body shape from age 5 to 50 were identified: lean-stable, lean-moderate increase, lean-marked increase, medium-stable/increase, and heavy-stable/increase. The lean-stable group was used as the reference. Among never smokers, the multivariable adjusted hazard ratio for death from any cause was 1.08 (95\% confidence interval 1.02 to 1.14 ) for women and 0.95 ( 0.88 to 1.03 ) for men in the lean-moderate increase group, 1.43 (1.33 to 1.54) for women and 1.11 (1.02 to 1.20) for men in the leanmarked increase group, 1.04 (0.97 to 1.12) for women and 1.01 (0.94 to 1.09) for men in the medium-stable/ increase group, and 1.64 (1.49 to 1.81) for women and 1.19 (1.08 to 1.32) for men in the heavy-stable/increase group. For cause specific mortality, participants in the heavy-stable/increase group had the highest risk, with

\section{WHAT IS ALREADY KNOWN ON THIS TOPIC}

Although high body mass index in adulthood has been linked to increased risk of mortality, the relation between body size over the life course and mortality is still unknown

\section{WHAT THIS STUDY ADDS}

This work represents the first effort to systematically assess the association of body shape throughout early and middle life with mortality risk by using a life course approach

Heavy body shape from age 5 up to 50 , especially an increase in middle life, was associated with higher mortality; in contrast, people who maintained a stably lean body shape had the lowest mortality

These results indicate the importance of weight management across the lifespan for health benefit

a hazard ratio among never smokers of 2.30 (1.88 to 2.81) in women and 1.45 (1.23 to 1.72) in men for cardiovascular disease, 1.37 (1.14 to 1.65$)$ in women and 1.07 (0.89 to 1.30) in men for cancer, and 1.59 (1.38 to 1.82 ) in women and 1.10 (0.95 to 1.29$)$ in men for other causes. The trajectory-mortality association was generally weaker among ever smokers than among never smokers (for all cause mortality: $P$ for interaction $<0.001$ in women and 0.06 in men). When participants were classified jointly according to trajectories and history of type 2 diabetes, the increased risk of death associated with heavier body shape trajectories was more pronounced among participants with type 2 diabetes than those without diabetes, and those in the heavy-stable/increase trajectory and with a history of diabetes had the highest risk of death.

\section{CONCLUSIONS}

Using the trajectory approach, we found that heavy body shape from age 5 up to 50 , especially the increase in middle life, was associated with higher mortality. In contrast, people who maintained a stably lean body shape had the lowest mortality. These results indicate the importance of weight management across the lifespan.

\section{Introduction}

The rising prevalence of obesity has become a public health crisis in the United States during the past three decades. Between 1988 and 2012, prevalence of obesity increased from $10 \%$ to $17 \%$ in children and adolescents aged 2-19 years and from $23 \%$ to $35 \%$ in adults aged 20 years or older. ${ }^{1-3}$ Substantial data indicate tracking of childhood weight status into adulthood. ${ }^{4}$ Overweight children had a greater than twofold risk of becoming overweight adults compared with normal weight children. Therefore, a life course perspective is crucial to better understanding of the health consequences of overweight and obesity and to development of effective prevention strategies targeting the life period at which excess body weight has a predominant influence on future risk of disease or death.

Many studies have assessed the association between adult obesity, often assessed by body mass index (BMI), and risk of premature death. ${ }^{5-7} \mathrm{~A}$ recent meta-analysis of 97 studies using standard BMI categories reported that relative to normal weight (BMI 18.5-<25), all cause mortality was increased for obesity (BMI $\geq 30$ ) but was decreased for overweight (BMI 25-29). ${ }^{8}$ These data are far from conclusive owing to methodological limitations, particularly reverse causation by pre-existing illness and confounding by smoking, which may have 
contributed to the inverse association between overweight and mortality. ${ }^{9}$

In contrast, the association of childhood or adolescent adiposity with adult mortality has been relatively less studied. Although high BMI in early life has been linked to increased mortality in most studies, whether this link is independent of adulthood BMI remains unclear. ${ }^{10-15}$ This question is challenging to answer, because adulthood BMI is likely to be on the causal path from childhood BMI to later disease. ${ }^{12}$ Statistical adjustment for adulthood BMI may introduce over-adjustment bias and make interpreting results difficult. Other techniques, such as analysis of different combinations of BMI groups (for example, obese versus non-obese) characterized over the life course, can result in loss of information due to discrete categorization. ${ }^{16}$

Therefore, to extend our knowledge, we used a different, trajectory based approach to assess the relation between body shape in early and middle life and risk of all cause and cause specific mortality in two large US cohort studies. By classifying participants into distinct, mutually exclusive trajectory groups, our study allows a close scrutiny of the population heterogeneity in change in body shape over the life course and permits direct comparison of the mortality risk across these groups.

\section{Methods}

\section{Study population}

The Nurses' Health Study (NHS) and Health Professionals Follow-up Study (HPFS) are two ongoing US cohorts that respectively enrolled 121700 registered female nurses aged 30-55 years in 1976 and 51529 male health professionals aged 40-75 years in 1986. Similar follow-up procedures have been used in the two cohorts. ${ }^{1718}$ Briefly, detailed questionnaires were administered at baseline enrollment and every two years thereafter to collect updated lifestyle and medical information. Diet was assessed using validated food frequency questionnaires every four years. The follow-up rates of the two cohorts had been $95.4 \%$ in the NHS and 95.9\% in the HPFS.

\section{Body shape assessment}

In 1988 participants in both cohorts were asked to choose one of the nine pictorial body diagrams (somatotypes) developed by Stunkard et al that best depicted their body shape at ages $5,10,20,30$, and 40 years. ${ }^{19}$ We have assessed the validity of this measure of body shape in early life among 181 participants aged 71-76 years in the Third Harvard Growth Study. ${ }^{20}$ We compared participants' recalled somatotypes with their measured BMI at approximately the same ages. The Pearson correlation coefficients for age 5, 10, and 20 were $0.60,0.65$, and 0.66 in women and $0.36,0.66$, and 0.53 in men. ${ }^{20}$

Participants reported their height on the baseline questionnaire and provided their current body weight on biennial follow-up questionnaires. We used these data to calculate their BMI at age 30,40 , and 50 . To minimize random variation, we used the average BMI at each of these ages plus/minus 3 years. For example, we used the average BMI from age 37 to 43 to represent the BMI for age 40. Because some participants were already at an older age at baseline, the BMI data were not complete in all participants (see supplementary methods). In addition, participants were asked to recall their body weight at age 18 in 1980 in the NHS and at age 21 in 1986 in the HPFS, as previously described. ${ }^{21}$ We used these data to calculate the BMI at adolescence.

Because the somatotype data were queried up to age 40 , to better characterize the trajectory of body shape from early through middle life we used participants' BMI at age 50 as a surrogate for their body shape at that age. To convert BMI to the same scale as somatotypes (ranging from 1 to 9), we used a proportional odds mixed effects model by linking the BMI to the somatotype data at younger ages and then used this model to predict the somatotype at age 50 on the basis of the BMI at that age. More details about model development and prediction are provided in the supplementary methods.

\section{Ascertainment of death}

We identified deaths from state statistics records, the National Death Index, next of kin, and the postal system. ${ }^{22}$ Cause of death was identified from death certificates or review of medical records by physicians. For this analysis, we assessed all cause mortality and death from cardiovascular disease (ICD-8 (international classification of diseases, eighth revision), codes 390 to 458), coronary heart disease (codes 390 to 429, 440 to 458), stroke (codes 430 to 438), cancer (codes 140 to 207 (colorectum 153, 154; pancreas 157; breast 174; endometrium 182; prostate 185)), and other causes.

\section{Statistical analysis}

Among participants who provided somatotype data for at least four different ages, we excluded those who died or had a history of diabetes, cardiovascular disease, or cancer before age 50. We included a total of 81477 women from the NHS and 37163 men from the HPFS in the trajectory analysis (see flowchart in supplementary figure A). We used a group based modeling approach implemented by SAS Proc Traj to identify subgroups within each cohort that shared a similar underlying trajectory of body shape from age 5 up to $50 .{ }^{2324}$ Details about trajectory analysis have been described elsewhere. ${ }^{25}$ Briefly, the longitudinal body shape data were fitted by maximum likelihood method as a mixture of multiple latent trajectories in a censored normal model with a polynomial function of age. ${ }^{24}$ We used the bayesian information criterion to determine the optimal number and shapes of trajectory groups through a two stage approach. ${ }^{26}$ The model with five trajectories and a cubic function of age showed the best fit to the data. We named the trajectories on the basis of the visual pattern of change in body shape over age and estimated the mean body shape levels for each trajectory at each age from the final model. We then calculated the posterior predicted probability for each participant of being a member of each of the five trajectories and assigned participants into the trajectory to which their posterior 
probability of membership was greatest. The average posterior probability for each trajectory was $0.91,0.97$, $0.85,0.87$, and 0.91 in women and $0.85,0.92,0.88,0.88$, and 0.93 in men, indicating a good discrimination of our trajectory assignment.

We then calculated the 15 year risk of death in each of the trajectory groups by using the product limit method and assessed the association of trajectories with all cause and cause specific mortality. To minimize the influence of reverse causation arising from undiagnosed illness induced weight loss, we allowed for a 10 year lag period; thus follow-up time was calculated from age 60 to the age of death or the end of the study period (1 June 2012 for the NHS and 31 January 2012 for the HPFS), whichever came first. A total of 80266 women and 36622 men contributing 1300351 and 541066 person years through a median of 15 and 16 years of follow-up, respectively, were included in the primary analysis. We used Cox proportional hazards model to estimate hazard ratios and 95\% confidence intervals of death in relation to trajectory groups, using the lean-stable group as the reference. We adjusted for several potential predictors for survival to control for confounding, and more details about covariate assessment are described in the supplementary methods. We did stratified analysis according to smoking status. Given the critical role of obesity in type 2 diabetes that may further increase risk of cardiovascular disease and cancer, we also examined the joint association of trajectory and history of type 2 diabetes with mortality. Because participants with a history of diabetes before age 50 had already been excluded, this analysis included only diabetes that was diagnosed after age 50 and treated it as a time varying variable. We used a likelihood ratio test to calculate $\mathrm{P}$ values for interaction by smoking and history of diabetes.

We used SAS 9.4 for all analyses. All statistical tests were two sided, and we considered $\mathrm{P}<0.05$ to be statistically significant.

\section{Patient involvement}

No patients were involved in setting the research question or the outcome measures, nor were they involved in the design and implementation of the study. There are no plans to involve patients in dissemination.

\section{Results}

Through group based trajectory modeling, we identified five distinct trajectories of body shape from age 5 to 50 among 80266 women and 36622 men. Figure 1 shows the estimated mean body shape levels in the five trajectories at each age: 35\% (28302) of women and 25\% (9086) of men maintained a lean, albeit slightly increasing, body shape throughout life (lean-stable group); $29 \%$ (23013) of women and 17\% (6221) of men started lean and then experienced a moderate increase in body shape (lean-moderate increase group); 11\% (8815) of women and 17\% (6241) of men started lean and then gained a substantial amount of weight (lean-marked increase group); 19\% (15475) of women and 28\% (10164) of men started with a medium body shape and then maintained or gained some weight over time (medium-stable/increase group); and 6\% (4661) of women and 13\% (4910) of men started heavy and then maintained or gained weight (heavy-stable/increase group).

As shown in table 1, BMI in each trajectory tracked well from adolescence to late adulthood. For example, in women, the mean BMI in the lean-stable group remained below 24 over the life course, whereas the mean BMI in the lean-marked increase group increased from 22.8 to 31.0 between age 18 and 50. We also noted that participants in the five trajectories showed distinctive lifestyle patterns: those in the lean-stable and medium-stable/increase groups were more physically active, tended to use multivitamins, and consumed a healthier diet than those in the other groups.
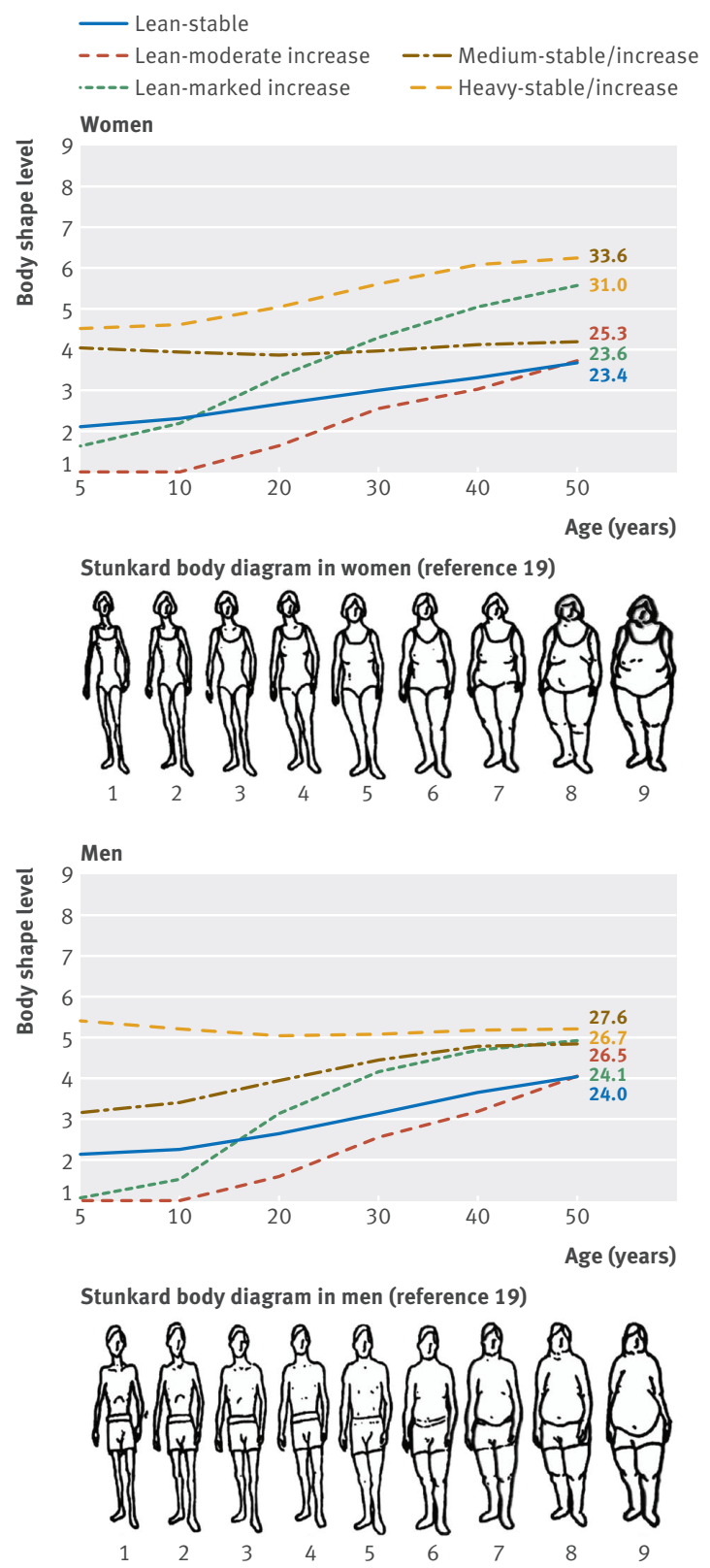

Fig 1 | Trajectories of body shape by age in women (top) and men (bottom). Mean body shape levels on y axis were estimated from trajectory models. Mean body mass index (BMI) at age 50 is shown for each trajectory group 


\begin{tabular}{|c|c|c|c|c|c|}
\hline \multirow[b]{2}{*}{ Variable } & \multicolumn{5}{|c|}{ Body shape trajectory group } \\
\hline & Lean-stable & $\begin{array}{l}\text { Lean-moderate } \\
\text { increase }\end{array}$ & $\begin{array}{l}\text { Lean-marked } \\
\text { increase }\end{array}$ & $\begin{array}{l}\text { Medium-stable/ } \\
\text { increase }\end{array}$ & $\begin{array}{l}\text { Heavy-stable/ } \\
\text { increase }\end{array}$ \\
\hline \multicolumn{6}{|l|}{ Women } \\
\hline No (\%) participants & $28302(35)$ & $23013(29)$ & 8815 (11) & $15475(19)$ & $4661(6)$ \\
\hline BMI at age $18+$ & $20.6(2.0)$ & $19.6(1.9)$ & $22.8(2.9)$ & $22.7(2.6)$ & $26.3(3.9)$ \\
\hline $\mathrm{BMI}$ at age $40 \dagger$ & $22.0(2.2)$ & $22.0(2.6)$ & $28.4(3.9)$ & $23.9(2.7)$ & $31.3(5.0)$ \\
\hline BMI at age $50 \dagger$ & $23.4(2.8)$ & $23.6(3.1)$ & $31.0(4.6)$ & $25.3(3.4)$ & $33.6(5.7)$ \\
\hline Height, inches & $64.5(2.4)$ & $64.5(2.5)$ & $64.4(2.5)$ & $64.5(2.4)$ & $64.7(2.5)$ \\
\hline Physical activity, METs-hours/week & $17.6(19.0)$ & $16.5(18.3)$ & $12.7(15.9)$ & $16.5(16.9)$ & $12.5(14.4)$ \\
\hline Alcohol consumption, g/day $\ddagger$ & $6.9(9.8)$ & $6.1(9.3)$ & $4.1(7.8)$ & $6.7(9.7)$ & $3.9(7.6)$ \\
\hline Alternative Healthy Eating Index $¥$ & $44.7(8.9)$ & $43.7(8.8)$ & $42.9(8.5)$ & $45.3(8.9)$ & $43.6(8.8)$ \\
\hline Pack years of smoking§ & $24.6(20.7)$ & $24.1(20.3)$ & $24.6(21.5)$ & $25.8(21.1)$ & $26.3(22.1)$ \\
\hline Smoking status, № (\%): & 28138 & 22881 & 8763 & 15397 & 4619 \\
\hline Never & $12441(44)$ & $10547(46)$ & $4258(49)$ & $6159(40)$ & $1969(42)$ \\
\hline Past & $11459(41)$ & $8918(40)$ & $3559(40)$ & $6772(44)$ & $2082(45)$ \\
\hline Current & $4238(15)$ & $3416(14)$ & $946(11)$ & $2466(16)$ & $568(13)$ \\
\hline No (\%) multivitamin use & $15008(53)$ & $11307(49)$ & $4406(50)$ & $8115(52)$ & $2393(51)$ \\
\hline \multicolumn{6}{|l|}{ Men } \\
\hline No (\%) participants & $9086(25)$ & $6221(17)$ & $6241(17)$ & $10164(28)$ & $4910(13)$ \\
\hline BMI at age $21 \dagger$ & $21.0(4.6)$ & $20.3(4.5)$ & $22.1(5.2)$ & $22.8(5.3)$ & $24.3(6.0)$ \\
\hline $\mathrm{BMI}$ at age $40 \dagger$ & $23.2(1.9)$ & $23.2(2.6)$ & $25.5(2.9)$ & $25.6(2.6)$ & $26.6(3.2)$ \\
\hline $\mathrm{BMI}$ at age $50+$ & $24.1(2.3)$ & $24.0(2.6)$ & $26.7(3.1)$ & $26.5(2.9)$ & $27.6(3.7)$ \\
\hline Height, inches & $70.2(2.6)$ & $70.3(2.7)$ & $70.1(2.7)$ & $70.0(2.6)$ & $70.1(2.6)$ \\
\hline Physical activity, METs-hours/weekł & $28.2(29.7)$ & $26.2(25.9)$ & $26.0(27.6)$ & $28.0(28.0)$ & $28.1(27.9)$ \\
\hline Alcohol consumption, g/day $\ddagger$ & $11.4(14.4)$ & $11.5(14.1)$ & $11.7(15.1)$ & $11.5(15.0)$ & $11.1(14.3)$ \\
\hline Alternative Healthy Eating Index $\ddagger$ & $41.7(9.8)$ & $41.3(9.7)$ & $40.7(9.6)$ & $41.6(9.4)$ & $42.3(9.6)$ \\
\hline Pack-years of smoking§ & $25.3(19.7)$ & $25.5(19.3)$ & $26.4(20.3)$ & $26.2(20.2)$ & $28.0(21.4)$ \\
\hline Smoking status, No (\%): & 8762 & 6010 & 6000 & 9768 & 4725 \\
\hline Never & $4429(51)$ & $2656(46)$ & $2642(45)$ & $4628(47)$ & $2035(42)$ \\
\hline Past & $3781(43)$ & $2940(48)$ & $2915(48)$ & $4471(46)$ & $2344(50)$ \\
\hline Current & $552(6)$ & $414(6)$ & $443(7)$ & $669(7)$ & $346(8)$ \\
\hline No (\%) multivitamin use & $4498(50)$ & $2937(47)$ & $2942(47)$ & $4986(49)$ & $2458(50)$ \\
\hline
\end{tabular}

$\mathrm{BMI}=$ body mass index; MET=metabolic equivalent of task.

*All variables are standardized by age at baseline (1976 for women in Nurses' Health Study and 1986 for men in Health Professionals Follow-up Study). Owing to large sample size, P values for testing difference across trajectory groups were $<0.001$ for all variables listed.

tData were not available in all participants because some participants were older than 40 or 50 at baseline or did not provide their body weight at age 18 or 21

$\ddagger$ Cumulative average measurements from baseline up to age 50 years.

$\S$ Among ever smokers only.

Table 2 shows the associations between trajectories and mortality in women. Overall, women in the other four trajectory groups all had a higher all cause mortality than those in the lean-stable group. After stratification by smoking, the association seemed to be much stronger among never smokers than among ever smokers ( $P$ for interaction $<0.001)$. Among never smokers, the multivariable adjusted hazard ratio for death from any cause was 1.08 (95\% confidence interval 1.02 to 1.14 ) for the lean-moderate increase group, 1.43 (1.33 to 1.54) for the lean-marked increase group, 1.04 (0.97 to 1.12) for the medium-stable/increase group, and 1.64 (1.49 to 1.81) for the heavy-stable/increase group. For specific causes of death among never smokers, we found a strong positive association for cardiovascular disease, especially coronary heart disease, with hazard ratios ranging from 1.13 to 2.55 across trajectories. Mortality from stroke, cancer, and other causes was also increased for the lean-marked increase and heavy-stable/increase groups; the latter group had the highest risk (hazard ratio ranged from 1.37 to 1.82 ).
In men, compared with the lean-stable group, the risk of death was increased for the lean-marked increase (hazard ratio 1.14, 1.08 to 1.20 ), medium-stable/increase (1.06, 1.01 to 1.11), and heavy-stable/ increase groups $(1.16,1.10$ to 1.23$)$ (table 3$)$. As in women, the association for the heavy-stable/increase group was stronger among never smokers than ever smokers ( $\mathrm{P}$ for interaction=0.06). For cause specific mortality among never smokers, men in the leanmarked increase and heavy-stable/increase groups had higher risk of death from cardiovascular and other causes, with hazard ratios ranging from 1.05 to 1.45 . Interestingly, we found a stronger association with stroke mortality among ever smokers than never smokers ( $\mathrm{P}$ for interaction $=0.008$ ). We found no association for cancer mortality in either the overall or the smoking stratified analysis.

To test the robustness of our findings to participants with modest trajectory assignment, we excluded 14107 women and 8019 men whose trajectory assignment probability was below 0.80 . Despite the compromised 


\begin{tabular}{|c|c|c|c|c|c|c|}
\hline \multirow[b]{2}{*}{ Cause of death } & \multicolumn{5}{|c|}{ Body shape trajectory group } & \multirow[b]{2}{*}{$\begin{array}{l}\text { P for interaction } \\
\text { with smoking } t\end{array}$} \\
\hline & Lean-stable & $\begin{array}{l}\text { Lean-moderate } \\
\text { increase }\end{array}$ & $\begin{array}{l}\text { Lean-marked } \\
\text { increase }\end{array}$ & $\begin{array}{l}\text { Medium-stable/ } \\
\text { increase }\end{array}$ & $\begin{array}{l}\text { Heavy-stable/ } \\
\text { increase }\end{array}$ & \\
\hline \multicolumn{7}{|l|}{ All cause } \\
\hline No of deaths $(n=20415)$ & 6690 & 6199 & 2403 & 3717 & 1406 & \multirow{5}{*}{$<0.001$} \\
\hline 15 year risk of death, $\%$ & 11.8 & 12.4 & 16.7 & 12.7 & 19.7 & \\
\hline All & 1 (reference) & 1.05 (1.01 to 1.09$)$ & 1.29 (1.23 to 1.35$)$ & 1.05 (1.01 to 1.09$)$ & 1.48 (1.39 to 1.57$)$ & \\
\hline Never smokers & 1 (reference) & $1.08(1.02$ to 1.14$)$ & $1.43(1.33$ to 1.54$)$ & 1.04 (0.97 to 1.12$)$ & $1.64(1.49$ to 1.81$)$ & \\
\hline Ever smokers & 1 (reference) & 1.04 (0.99 to 1.08$)$ & 1.21 (1.14 to 1.28$)$ & 1.05 (1.00 to 1.10$)$ & 1.40 (1.30 to 1.50$)$ & \\
\hline \multicolumn{7}{|l|}{ Cardiovascular disease } \\
\hline No of deaths $(n=3953)$ & 1176 & 1183 & 565 & 676 & 353 & \\
\hline 15 year risk of death, $\%$ & 2.1 & 2.5 & 4.2 & 2.3 & 5.7 & \\
\hline All & 1 (reference) & $1.10(1.01$ to 1.19$)$ & 1.65 (1.49 to 1.82$)$ & 1.08 (0.99 to 1.19$)$ & $2.00(1.77$ to 2.25$)$ & \multirow{3}{*}{0.16} \\
\hline Never smokers & 1 (reference) & 1.24 (1.08 to 1.42$)$ & $1.77(1.51$ to 2.09$)$ & $1.13(0.95$ to 1.33$)$ & 2.30 (1.88 to 2.81$)$ & \\
\hline Ever smokers & 1 (reference) & 1.02 (0.92 to 1.13$)$ & 1.58 (1.39 to 1.80$)$ & 1.06 (0.94 to 1.19$)$ & 1.85 (1.59 to 2.15$)$ & \\
\hline \multicolumn{7}{|l|}{ Coronary heart disease } \\
\hline No of deaths $(n=2771)$ & 788 & 797 & 446 & 476 & 264 & \\
\hline 15 year risk of death, $\%$ & 1.5 & 1.8 & 3.5 & 1.6 & 4.4 & \\
\hline All & 1 (reference) & $1.10(1.00$ to 1.22$)$ & 1.91 (1.70 to 2.15$)$ & 1.14 (1.01 to 1.27$)$ & 2.17 (1.89 to 2.50$)$ & \multirow{3}{*}{0.15} \\
\hline Never smokers & 1 (reference) & 1.27 (1.08 to 1.49$)$ & 1.96 (1.61 to 2.38$)$ & 1.16 (0.95 to 1.42$)$ & 2.55 (2.01 to 3.23$)$ & \\
\hline Ever smokers & 1 (reference) & $1.02(0.90$ to 1.15$)$ & 1.90 (1.64 to 2.20$)$ & 1.12 (0.98 to 1.28$)$ & 2.00 (1.68 to 2.38$)$ & \\
\hline \multicolumn{7}{|l|}{ Stroke } \\
\hline No of deaths ( $n=1182$ ) & 388 & 386 & 119 & 200 & 89 & \\
\hline 15 year risk of death, $\%$ & 0.6 & 0.7 & 0.7 & 0.6 & 1.4 & \\
\hline All & 1 (reference) & 1.08 (0.94 to 1.25$)$ & 1.09 (0.88 to 1.34$)$ & 0.98 (0.83 to 1.16$)$ & $1.62(1.28$ to 2.04$)$ & \multirow{3}{*}{0.31} \\
\hline Never smokers & 1 (reference) & 1.18 (0.94 to 1.49$)$ & 1.41 (1.04 to 1.91$)$ & 1.06 (0.79 to 1.43$)$ & $1.82(1.24$ to 2.66$)$ & \\
\hline Ever smokers & 1 (reference) & 1.03 (0.86 to 1.23$)$ & 0.89 (0.67 to 1.18$)$ & $0.93(0.76$ to 1.15$)$ & $1.52(1.13$ to 2.03$)$ & \\
\hline \multicolumn{7}{|l|}{ Cancer } \\
\hline No of deaths $(n=6744)$ & 2284 & 2075 & 708 & 1273 & 404 & \\
\hline 15 year risk of death, $\%$ & 6.0 & 6.3 & 7.1 & 6.3 & 8.0 & \\
\hline All & 1 (reference) & 1.04 (0.98 to 1.10$)$ & 1.13 (1.04 to 1.23$)$ & $1.03(0.97$ to 1.11$)$ & 1.24 (1.11 to 1.38$)$ & \multirow{3}{*}{0.27} \\
\hline Never smokers & 1 (reference) & 1.04 (0.93 to 1.15$)$ & 1.26 (1.09 to 1.44$)$ & $1.08(0.95$ to 1.23$)$ & 1.37 (1.14 to 1.65$)$ & \\
\hline Ever smokers & 1 (reference) & 1.04 (0.96 to 1.11$)$ & 1.07 (0.96 to 1.19$)$ & 1.02 (0.94 to 1.10$)$ & $1.18(1.04$ to 1.34$)$ & \\
\hline \multicolumn{7}{|l|}{ Other causes } \\
\hline No of deaths $(\mathrm{n}=9718)$ & 3230 & 2941 & 1130 & 1768 & 649 & \\
\hline 15 year risk of death, \% & 4.1 & 4.2 & 6.4 & 4.8 & 7.5 & \\
\hline All & 1 (reference) & 1.04 (0.99 to 1.09$)$ & 1.27 (1.19 to 1.37$)$ & 1.04 (0.99 to 1.11$)$ & 1.45 (1.33 to 1.58$)$ & \multirow{3}{*}{0.003} \\
\hline Never smokers & 1 (reference) & 1.04 (0.96 to 1.13$)$ & 1.44 (1.29 to 1.59$)$ & 0.99 (0.89 to 1.09 ) & 1.59 (1.38 to 1.82$)$ & \\
\hline Ever smokers & 1 (reference) & 1.04 (0.97 to 1.11$)$ & $1.17(1.06$ to 1.28$)$ & 1.07 (1.00 to 1.15$)$ & $1.38(1.24$ to 1.54$)$ & \\
\hline \multicolumn{7}{|c|}{$\begin{array}{l}\text { *Follow-up started at age } 60 \text {. Hazard ratios were estimated from age stratified Cox proportional hazards model adjusted for age (continuous), height (continuous), race (non-white or white), } \\
\text { pack years of smoking }(0,1-6,6-\leq 20 \text {, or }>20) \text {, family history of cancer (yes or no), history of lower gastrointestinal endoscopy (yes or no; for analysis of total cancer), multivitamin use (yes or } \\
\text { no), regular aspirin/non-steroidal anti-inflammatory drug use (yes or no), history of physical examination (yes and for screening, yes and for symptoms, or no), mammography (yes and for } \\
\text { screening, yes and for symptoms, or no; for analysis of total cancer), menopausal hormone therapy (past use, current use, or no), physical activity (in fifths), alcohol consumption ( } 0-<0.5 \text {, } \\
0.5-<2,2-<8, \text { or } \geq 8 \mathrm{~g} / \text { day), and Alternate Healthy Eating Index dietary score (in fifths). } \\
+ \text { thikelihood ratio test with four degrees of freedom was used to compare model with product terms between smoking (binary: ever } v \text { never smoking) and trajectory groups (indicator variables } \\
\text { for four non-reference groups) against model without these terms. }\end{array}$} \\
\hline
\end{tabular}

statistical power, the trajectory-mortality associations were similar (supplementary table A).

We then assessed major cancers that have been associated with obesity (supplementary table B). Again, the trajectory-mortality associations were generally stronger among never smokers. Compared with the lean-stable group, the hazard ratio for postmenopausal breast cancer was 1.16 (1.00 to 1.35$)$ for the lean-moderate increase group, 1.06 (0.84 to 1.32$)$ for the lean-marked increase group, 0.90 (0.74 to 1.08) for the medium-stable/increase group, and 0.79 ( 0.56 to 1.11) for the heavy-stable/increase group. The heavy-stable/increase group had a higher mortality from cancers in the endometrium (hazard ratio 3.15, 1.96 to 5.07 ), pancreas (women: $2.15,1.49$ to 3.12 ; men:
$1.15,0.79$ to 1.66 ), and colorectum (women: $1.42,0.99$ to 2.03; men: 1.06, 0.74 to 1.53), although some of the confidence intervals contained one. We found no association for prostate cancer.

We further examined the joint associations of trajectory and history of diabetes with mortality among never smokers. Because the medium-stable/increase group had similar mortality to the lean-moderate increase group, we combined these two groups to maximize statistical power. Also, because the association patterns were largely similar in the two cohorts (supplementary table C), we presented the pooled results for women and men by using the random effects meta-analysis approach. ${ }^{27}$ As shown in figure 2 , the risk of death from any cause, cardiovascular 


\begin{tabular}{|c|c|c|c|c|c|c|}
\hline \multirow[b]{2}{*}{ Cause of death } & \multicolumn{5}{|c|}{ Body shape trajectory group } & \multirow[b]{2}{*}{$\begin{array}{l}\text { P for interaction } \\
\text { with smokingt }\end{array}$} \\
\hline & Lean-stable & $\begin{array}{l}\text { Lean-moderate } \\
\text { increase }\end{array}$ & $\begin{array}{l}\text { Lean-marked } \\
\text { increase }\end{array}$ & $\begin{array}{l}\text { Medium-stable/ } \\
\text { increase }\end{array}$ & $\begin{array}{l}\text { Heavy-stable/ } \\
\text { increase }\end{array}$ & \\
\hline \multicolumn{7}{|l|}{ All cause } \\
\hline No of deaths ( $n=14943)$ & 3541 & 2958 & 2982 & 3723 & 1739 & \multirow{5}{*}{0.06} \\
\hline 15 year risk of death, $\%$ & 20.3 & 22.2 & 27.2 & 22.4 & 24.1 & \\
\hline All & 1 (reference) & 1.03 (0.98 to 1.08$)$ & 1.14 (1.08 to 1.20$)$ & 1.06 (1.01 to 1.11$)$ & 1.16 (1.10 to 1.23$)$ & \\
\hline Never smokers & 1 (reference) & 0.95 (0.88 to 1.03$)$ & $1.11(1.02$ to 1.20$)$ & $1.01(0.94$ to 1.09$)$ & $1.19(1.08$ to 1.32$)$ & \\
\hline Ever smokers & 1 (reference) & $1.07(1.01$ to 1.14$)$ & 1.15 (1.09 to 1.23$)$ & 1.09 (1.03 to 1.16$)$ & $1.15(1.07$ to 1.23$)$ & \\
\hline \multicolumn{7}{|l|}{ Cardiovascular disease } \\
\hline No of deaths $(\mathrm{n}=4813)$ & 1036 & 959 & 1037 & 1210 & 571 & \multirow{5}{*}{0.02} \\
\hline 15 year risk of death, $\%$ & 6.8 & 8.4 & 11.4 & 8.2 & 9.6 & \\
\hline All & 1 (reference) & $1.11(1.02$ to 1.21$)$ & $1.32(1.21$ to 1.44$)$ & 1.19 (1.09 to 1.29$)$ & $1.32(1.19$ to 1.47$)$ & \\
\hline Never smokers & 1 (reference) & 0.98 (0.84 to 1.13$)$ & 1.32 (1.15 to 1.52$)$ & $1.10(0.96$ to 1.26$)$ & $1.45(1.23$ to 1.72$)$ & \\
\hline Ever smokers & 1 (reference) & $1.19(1.07$ to 1.33$)$ & $1.32(1.19$ to 1.48$)$ & $1.24(1.12$ to 1.38$)$ & $1.26(1.11$ to 1.44$)$ & \\
\hline \multicolumn{7}{|l|}{ Coronary heart disease } \\
\hline No of deaths $(n=3975)$ & 840 & 791 & 854 & 998 & 492 & \\
\hline 15 year risk of death, $\%$ & 5.6 & 6.9 & 9.5 & 6.5 & 8.1 & \\
\hline All & 1 (reference) & $1.13(1.02$ to 1.25$)$ & $1.33(1.21$ to 1.46$)$ & 1.19 (1.09 to 1.31$)$ & $1.37(1.22$ to 1.53$)$ & \multirow{3}{*}{0.09} \\
\hline Never smokers & 1 (reference) & $1.07(0.91$ to 1.27$)$ & 1.35 (1.15 to 1.59$)$ & 1.12 (0.96 to 1.31$)$ & $1.58(1.31$ to 1.90$)$ & \\
\hline Ever smokers & 1 (reference) & $1.16(1.03$ to 1.31$)$ & 1.31 (1.16 to 1.48$)$ & $1.23(1.10$ to 1.38$)$ & $1.27(1.10$ to 1.47$)$ & \\
\hline \multicolumn{7}{|l|}{ Stroke } \\
\hline No of deaths $(n=838)$ & 196 & 168 & 183 & 212 & 79 & \multirow{5}{*}{0.008} \\
\hline 15 year risk of death, \% & 1.2 & 1.6 & 1.9 & 1.6 & 1.3 & \\
\hline All & 1 (reference) & 1.03 (0.84 to 1.27$)$ & 1.25 (1.02 to 1.53$)$ & 1.11 (0.92 to 1.35$)$ & 1.00 (0.77 to 1.29$)$ & \\
\hline Never smokers & 1 (reference) & 0.63 (0.45 to 0.90$)$ & 1.16 (0.86 to 1.57$)$ & 0.93 (0.69 to 1.25$)$ & 0.79 (0.51 to 1.23$)$ & \\
\hline Ever smokers & 1 (reference) & $1.38(1.06$ to 1.79$)$ & $1.34(1.02$ to 1.76$)$ & 1.28 (0.99 to 1.66$)$ & 1.17 (0.84 to 1.63$)$ & \\
\hline \multicolumn{7}{|l|}{ Cancer } \\
\hline No of deaths $(n=4098)$ & 1022 & 801 & 761 & 1022 & 492 & \\
\hline 15 year risk of death, $\%$ & 8.6 & 8.7 & 9.5 & 8.8 & 8.7 & \\
\hline All & 1 (reference) & 0.96 (0.88 to 1.06$)$ & $1.01(0.92$ to 1.11$)$ & 0.99 (0.91 to 1.08$)$ & 1.07 (0.96 to 1.19$)$ & \multirow{3}{*}{0.27} \\
\hline Never smokers & 1 (reference) & $1.03(0.88$ to 1.21$)$ & 0.99 (0.84 to 1.16$)$ & $0.92(0.79$ to 1.06$)$ & $1.07(0.89$ to 1.30$)$ & \\
\hline Ever smokers & 1 (reference) & 0.93 (0.83 to 1.04$)$ & 1.01 (0.90 to 1.14$)$ & 1.03 (0.93 to 1.15$)$ & 1.07 (0.94 to 1.22$)$ & \\
\hline \multicolumn{7}{|l|}{ Other causes } \\
\hline No of deaths $(n=6032)$ & 1483 & 1198 & 1184 & 1491 & 676 & \\
\hline 15 year risk of death, $\%$ & 6.5 & 6.9 & 9.2 & 7.3 & 8.0 & \\
\hline All & 1 (reference) & $1.02(0.94$ to 1.10$)$ & $1.10(1.02$ to 1.19$)$ & 1.02 (0.95 to 1.09$)$ & 1.10 (1.01 to 1.21$)$ & \multirow{3}{*}{0.09} \\
\hline Never smokers & 1 (reference) & 0.90 (0.79 to 1.02$)$ & 1.05 (0.93 to 1.19$)$ & 1.01 (0.90 to 1.13$)$ & $1.10(0.95$ to 1.29$)$ & \\
\hline Ever smokers & 1 (reference) & $1.10(0.99$ to 1.21$)$ & $1.13(1.02$ to 1.25$)$ & 1.02 (0.93 to 1.12$)$ & $1.10(0.98$ to 1.24$)$ & \\
\hline
\end{tabular}

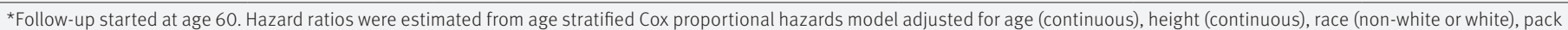
years of smoking $(0,1-<5,5-\leq 25,26-\leq 45$, or $>45)$, family history of cancer (yes or no), history of lower gastrointestinal endoscopy (yes or no; for analysis of total cancer), multivitamin use (yes or

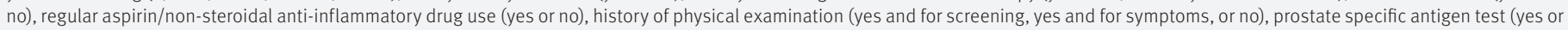
no; for analysis of total cancer), physical activity (in fifths), alcohol consumption $(0-<5,5-<10,10-<15,15-<30$, or $\geq 30 \mathrm{~g} /$ day), and Alternate Healthy Eating Index dietary score (in fifths). tLikelihood ratio test with four degrees of freedom was used to compare model with product terms between smoking (binary: ever $v$ never smoking) and trajectory groups (indicator variables for four non-reference groups) against model without these terms.

disease, and cancer all generally increased in a gradual manner from the lean-stable, lean/medium-moderate increase, lean-marked increase, to the heavy-stable/increase groups among the stratums with or without history of diabetes. However, the increase was more pronounced among participants with type 2 diabetes ( $\mathrm{P}$ for interaction $0.15,0.49$, and 0.01 for each of the three outcomes). Compared with participants who were in the lean-stable group and not diagnosed as having type 2 diabetes, those in the heavy-stable/increase trajectory who also had a history of diabetes were at the highest risk, and the multivariable adjusted hazard ratio was 1.84 (1.11 to 3.04) for all cause mortality, 2.60 (1.76 to 3.77 ) for cardiovascular mortality, and 2.09 (1.05 to 4.16) for cancer mortality.

\section{Discussion}

To our knowledge, this is the first study to investigate adiposity throughout early and middle life in relation to mortality. We used a trajectory approach to identify distinct subgroups of participants with similar evolution of body shape over the life course in two large cohort studies with standardized data collection for two to three decades. By comparing mortality risk between trajectory subgroups, we found that participants who remained heavy from age 5 to 50 had the highest risk of death, whereas those who maintained a stably lean body shape had the lowest mortality. Compared with the latter group, even those who were lean in childhood or adolescence but gained weight in middle life were at higher risk of mortality. This increased risk was more striking among never smokers than among ever 

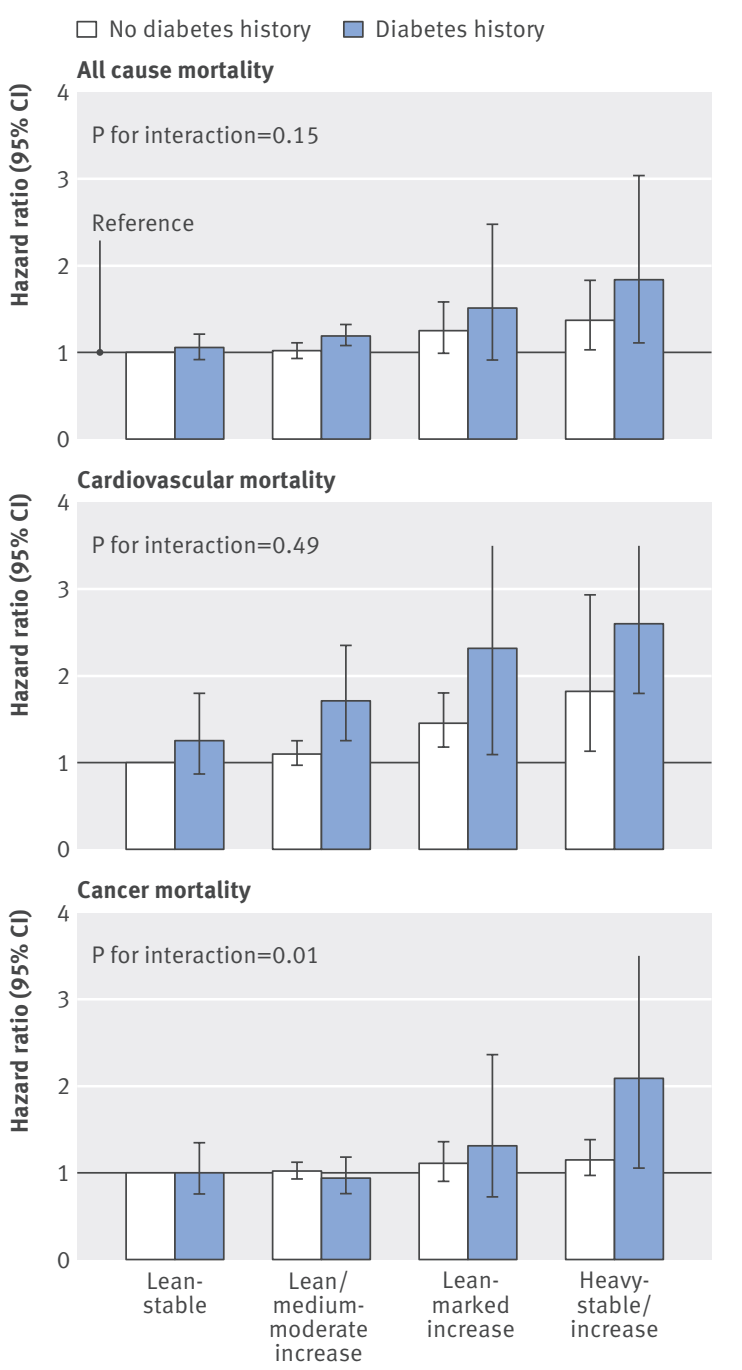

Fig 2 | Joint association of trajectories of body shape and history of type 2 diabetes with risk of all cause (top), cardiovascular (middle), and cancer mortality (bottom) among never smokers. Lean-moderate increase and medium-stable/increase groups were combined as "lean/medium-moderate increase" group owing to small number of cases. Multivariable Cox proportional hazards model was used to calculate hazard ratio within each cohort after adjustment for same set of covariates as in tables 2 and 3. Participants were categorized into eight groups according to trajectories and history of type 2 diabetes, with those in lean-stable group and without history of type 2 diabetes as reference. Pooled results from both cohorts are shown here. $P$ values for interaction were calculated from likelihood ratio test with three degrees of freedom by comparing model with product terms between diabetic history (binary) and trajectory groups (indicator variables for three non-reference groups) with model without these terms. P value for interaction for each individual trajectory group was also calculated by Wald test. For all cause mortality, individual $P$ values for interaction were 0.28 for lean/medium-moderate increase group, 0.09 for lean-marked increase group, and 0.04 for heavy-stable/increase group; corresponding $\mathrm{P}$ values for cardiovascular mortality were $0.21,0.16$, and 0.59 and those for cancer mortality were $0.64,0.34$, and 0.01 smokers. Our findings provide further scientific rationale for recommendations of weight management, especially avoidance of weight gain in middle life, for long term health benefit.

\section{Comparison with other studies}

The unique advantage of our trajectory approach over traditional analysis is its ability to map the developmental course and classify people into distinct, mutually exclusive groups. This not only helps to conceptualize the growth pattern of body shape but also allows us to probe into the population heterogeneity in the susceptibility of change in body shape over the life course, which has great implications for future research. For example, further studies should examine the relative contributions of people's genetics and behaviors to their trajectory profiles. Such investigations will provide critical insights into tailored prevention strategies. For example, the heavy-stable/increase group may have a larger genetic component, whereas the lean-marked increase group may be more behaviorally oriented. If this is the case, public health strategies of behavioral change should be targeted toward the latter group. ${ }^{28}$

The continuous process of body growth makes it challenging to determine whether the mortality risk associated with early life obesity is due to obesity in this young age or to obesity tracking into adulthood. Most previous studies have attempted to answer this question by statistical adjustment for adulthood BMI when examining the relation between childhood or adolescent adiposity and mortality. Inconsistent findings have been reported using this approach: some studies found that the association between obesity in adolescents and higher mortality was explained by adulthood BMI, ${ }^{29}$ but others observed that the increased mortality in overweight and obese adolescents was, at least partly, independent of BMI in adulthood. ${ }^{163031}$ However, this approach may represent an over-adjustment, as adulthood BMI may be a mediator for the effect of adiposity at a young age on risk of disease in adulhoodt. ${ }^{12}$ Moreover, adjustment for adulthood BMI controls not only for adult body size but also for the change in body weight from young age to adulthood. Determining whether it is early life adiposity or weight gain that is associated with the outcome is therefore difficult. ${ }^{32}$

In contrast, instead of assessing early and late life adiposity separately, our trajectory approach respects the continuity of body growth and answers a more tangible question about the relative mortality of people who experienced different developmental courses of body shape. We found that, compared with those who maintained a lean body shape, people with a heavy body shape throughout life had a substantially increased mortality. Of note, the relative risk estimates were stronger in women than in men, although strict comparison between the two sexes is difficult because the trajectories were created within each cohort separately and sex specific reference groups were used in the analysis. Better performance of the pictograms in assessing body shape in women (see Methods) may 
have contributed to the stronger results than in men. Another explanation may be related to the much higher BMI in the heavy-stable/increase group in women than in men (for example, mean BMI at age 50: 33.6 v 27.6; table 1). More interestingly, we found that even people who were lean in early life but gained weight later were at higher risk of mortality, and the heavier the people became in middle adulthood the higher their risk was. These findings indicate a critical deleterious effect of weight gain in middle life, which has been associated with a range of cardiometabolic abnormalities, such as atherogenic lipid profile, insulin resistance, systemic inflammation, lower adiponectin, and higher leptin. ${ }^{33}$ Accordingly, weight gain from adolescence throughout adulthood has been linked to higher risks of diabetes, cardiovascular disease, and cancers in the colorectum, breast, and pancreas, as well as premature death. ${ }^{2134-37}$ In contrast, lifestyle based weight loss interventions have been shown to result in favorable changes in cardiometabolic risk factors and potentially lower mortality. ${ }^{38-40}$

Smoking is an important risk factor for many diseases and premature death, but smokers generally have a lower BMI than non-smokers. ${ }^{41}$ Thus, smoking may significantly confound and attenuate the association between body weight and mortality. ${ }^{42}$ Consistent with previous studies, ${ }^{543}$ we found that body shape trajectory was more strongly associated with mortality among never smokers than among ever smokers. This difference seemed to be more striking in women than in men, which may be a result of the higher proportion of current smokers among women than among men (14\% $v 7 \%$ ) in our cohorts. Moreover, some, albeit inconsistent, evidence suggests a greater metabolic influence of smoking in women than men and that women tend to gain more weight after smoking cessation than men. ${ }^{41445}$ Therefore, excluding smokers from the analysis may have a larger effect on the trajectory-mortality associations in women than in men. Intriguingly, for stroke, the trajectory-mortality association in men was stronger among ever smokers than among never smokers, especially for the lean-moderate or marked increase groups. Given the paucity of data on the smoking-obesity interaction with stroke mortality, further studies are needed to assess whether smoking may interact with weight gain to influence risk and progression of stroke.

Another methodological problem with examination of the obesity-mortality relation is related to reverse causation from pre-existing illnesses (for example, chronic obstructive pulmonary disease and undiagnosed cancer) that can result in weight loss and, at the same time, elevate patients' risk of death. Therefore, failing to tackle this problem may lead to an attenuated association or even a spurious inverse association between body weight and mortality. For example, this reverse causation phenomenon has been suggested to have contributed to the lower risk of mortality associated with overweight reported in the recent meta-analysis. ${ }^{8}$ Excluding participants with chronic conditions at baseline enrollment may help to abate, but hardly eliminates, this problem. In this study, we adopted a 10 year lag period for follow-up of death after assessment of trajectory to minimize reverse causation. We found that a heavy trajectory was associated with an increased risk of mortality even from causes other than cardiovascular disease or cancer (for example, respiratory or neurodegenerative disease), conditions that are likely to cause significant weight loss long before diagnosis in older people and are thus more sensitive to reverse causation.

We also observed an interaction between body shape trajectory and history of type 2 diabetes. People who had both a heavy body shape and a history of diabetes were at substantially higher risk of death than those who did not have either of the conditions. Diabetes is an important risk factor for cardiovascular disease. Increasing evidence also relates diabetes to a higher risk of cancer, including cancers of the pancreas, endometrium, liver, colorectum, and breast, possibly by mechanisms related to hyperinsulinema, hyperglycemia, and chronic inflammation. ${ }^{46}$ Furthermore, some studies found that diabetes may increase mortality in patients with cancer. ${ }^{47}$ Patients with cancer and pre-existing diabetes had a higher mortality than their non-diabetic counterparts, even after adjustment for BMI. 4849 Taken together, these data indicate that diabetes may influence risk of disease and death above and beyond the risk conferred by excess body weight.

\section{Strengths and limitations of study}

Our study takes advantage of the rigorous follow-up of two well established cohorts and assessed a wide range of causes of death in addition to overall mortality. The trajectory method we used also provides an attractive alternative to traditional analysis by integration of exposure data collected across the lifespan. ${ }^{25} 50$ Although this approach has previously been used to assess the association of obesity with mortality, these studies collected data only after age 50 and were thus unable to capture any effect that early life body shape may exert. ${ }^{5152}$ In addition, we took great care to minimize several methodological problems to provide solid evidence for the influence of body shape over the life course on mortality. Given the advancement of technology, especially the growing use of electronic medical records, ${ }^{53}$ trajectory assessment will conceivably have an improved accuracy and may not be an overwhelming task for clinical practitioners to perform in the future. Therefore, further studies are needed to determine whether assessing people's trajectory in clinical settings would produce significantly more information than just measuring BMI, by comparing clinically relevant measures (such as predictive capability) and also taking into account the cost associated with each tool.

Several limitations of the study need to be noted as well. Firstly, body shape assessed by recalled somatotype is subject to measurement error. However, given our prospective design, any error would have probably attenuated the observed association. Admittedly, a study to prospectively measure participants' body size across the lifespan and then follow them up for 
death would be ideal. Nevertheless, practical constraints probably make such study unfeasible. Secondly, a limited number of trajectories were derived that may not accurately reflect each person's profile of body shape. However, the good discrimination of our trajectory building model and well tracked change in BMI across trajectories indicate that these trajectories can parsimoniously summarize the predominant features of lifetime body shape in our population without a significant loss of information. Additionally, the fact that we obtained similar results after excluding participants with suboptimal trajectory assignment was reassuring. Thirdly, our study participants were all health professionals. However, the adiposity-mortality relation we observed is unlikely to differ substantially from that in the general population. On the other hand, the homogeneity of our study population helps to minimize the likelihood of uncontrolled confounding.

\section{Conclusions}

We found that heavy body shape throughout early and middle life, especially a weight increase in middle life, was associated with higher mortality. In contrast, people who maintained a stably lean body shape had the lowest mortality. These results indicate the health benefit of weight management across the lifespan.

MS is a trainee of the Harvard Transdisciplinary Research Center on Energetics and Cancer (TREC). We thank the participants and staff of the Nurses' Health Study and the Health Professionals Follow-up Study for their valuable contributions, as well as the following state cancer registries for their help: AL, AZ, AR, CA, CO, CT, DE, FL, GA, ID, IL, IN, IA, KY, LA, ME, MD, MA, MI, NE, NH, NJ, NY, NC, ND, OH, OK, OR, PA, RI, SC, TN, TX, VA, WA, and WY. The authors assume full responsibility for analysis and interpretation of these data.

Contributors: MS and ELG conceived and designed the study. FBH, KW, AM, ATC, WCW, and ELG acquired the data. MS and ELG drafted the manuscript. All the authors critically revised the manuscript for important intellectual content. MS did the statistical analysis. FBH, ATC, WCW, and ELG obtained funding. MS and ELG were responsible for administrative, technical, or material support. ELG was responsible for study supervision. MS and ELG had full access to all of the data in the study and take responsibility for the integrity of the data and the accuracy of the data analysis. MS is the guarantor.

Funding: This work was supported by the National Institutes of Health (UM1 CA186107, P01 CA87969, UM1 CA167552, 1U54CA155626, and K24 DK098311) and Boston Obesity Nutrition Research Center (DK46200). The funders had no role in design and conduct of the study; collection, management, analysis, and interpretation of the data; preparation, review, and approval of the manuscript; or the decision to submit the manuscript for publication.

Competing interests: All authors have completed the ICMJE uniform disclosure form at http://www.icmje.org/coi_disclosure.pdf and declare: no support from any organization for the submitted work other than those described above; no financial relationships with any organizations that might have an interest in the submitted work in the previous three years; no other relationships or activities that could appear to have influenced the submitted work.

Ethical approval: This investigation was approved by the Institutional Review Board at the Brigham and Women's Hospital and the Harvard T $\mathrm{H}$ Chan School of Public Health.

Transparency declaration: The lead author (the manuscript's guarantor) affirms that the manuscript is an honest, accurate, and transparent account of the study being reported; that no important aspects of the study have been omitted; and that any discrepancies from the study as planned (and, if relevant, registered) have been explained.

Data sharing: No additional data available.
This is an Open Access article distributed in accordance with the Creative Commons Attribution Non Commercial (CC BY-NC 3.0) license, which permits others to distribute, remix, adapt, build upon this work non-commercially, and license their derivative works on different terms, provided the original work is properly cited and the use is non-commercial. See: http://creativecommons.org/licenses/ by-nc/3.0/.

1 Fryar CD, Carroll MD, Ogden CL. Prevalence of overweight and obesity among children and adolescents: United States, 1963-1965 through 2011-2012. 2014. http://www.cdc.gov/nchs/data/hestat/ obesity_child_11_12/obesity_child_11_12.htm.

2 Ogden CL, Carroll MD, Kit BK, Flegal KM. Prevalence of childhood and adult obesity in the United States, 2011-2012. JAMA 2014;311:80614. doi:10.1001/jama.2014.732

3 Ogden CL, Carroll MD. Prevalence of overweight, obesity, and extreme obesity among adults: United States, trends 1960-1962 through 2007-2008. 2010. http://www.cdc.gov/NCHS/data/hestat/ obesity_adult_07_08/obesity_adult_07_08.pdf.

4 Singh AS, Mulder C, Twisk JW, van Mechelen W, Chinapaw MJ. Tracking of childhood overweight into adulthood: a systematic review of the literature. Obes Rev 2008;9:474-88. doi:10.1111/j.1467-789X.2008.00475.x.

5 Berrington de Gonzalez A, Hartge P, Cerhan JR, et al. Body-mass index and mortality among 1.46 million white adults. N Engl J Med 2010;363:2211-9. doi:10.1056/NEJMoa1000367.

6 Whitlock G, Lewington S, Sherliker P, et al. Prospective Studies Collaboration. Body-mass index and cause-specific mortality in 900 000 adults: collaborative analyses of 57 prospective studies. Lancet 2009;373:1083-96. doi:10.1016/S0140-6736(09)60318-4.

$7 \quad$ Zheng W, McLerran DF, Rolland B, et al. Association between body-mass index and risk of death in more than 1 million Asians. N Engl J Med 2011;364:719-29. doi:10.1056/NEJMoa1010679.

8 Flegal KM, Kit BK, Orpana H, Graubard BI. Association of all-cause mortality with overweight and obesity using standard body mass index categories: a systematic review and meta-analysis. JAMA 2013;309:71-82. doi:10.1001/jama.2012.113905.

9 Tobias DK, Hu FB. Does being overweight really reduce mortality?Obesity (Silver Spring) 2013;21:1746-9. doi:10.1002/ oby. 20602.

10 Gunnell DJ, Frankel SJ, Nanchahal K, Peters TJ, Davey Smith G. Childhood obesity and adult cardiovascular mortality: a 57-y follow-up study based on the Boyd Orr cohort. Am J Clin Nutr 1998:67:1111-8.pmid:9625081.

11 Ma J, Flanders WD, Ward EM, Jemal A. Body mass index in young adulthood and premature death: analyses of the US National Health Interview Survey linked mortality files. Am J Epidemiol 2011;174:934-44. doi:10.1093/aje/kwr169.

12 Park MH, Falconer C, Viner RM, Kinra S. The impact of childhood obesity on morbidity and mortality in adulthood: a systematic review. Obes Rev 2012;13:985-1000. doi:10.1111/j.1467-789X.2012.01015.x.

13 Neovius M, Sundström J, Rasmussen F. Combined effects of overweight and smoking in late adolescence on subsequent mortality: nationwide cohort study. BMJ 2009;338:b496. doi:10.1136/ bmib496.

14 Bjørge T, Engeland A, Tverdal A, Smith GD. Body mass index in adolescence in relation to cause-specific mortality: a follow-up of 230,000 Norwegian adolescents. Am J Epidemiol 2008;168:30-7. doi:10.1093/aje/kwn096.

15 Engeland A, Bjørge T, Søgaard AJ, Tverdal A. Body mass index in adolescence in relation to total mortality: 32-year follow-up of 227,000 Norwegian boys and girls. Am J Epidemiol 2003;157:517-23. doi:10.1093/aje/kwf219.

16 Hirko KA, Kantor ED, Cohen SS, Blot WJ, Stampfer MJ, Signorello LB Body mass index in young adulthood, obesity trajectory, and premature mortality. Am J Epidemiol 2015;182:441-50. doi:10.1093/ aje/kwv084

17 Rimm EB, Giovannucci EL, Willett WC, et al. Prospective study of alcohol consumption and risk of coronary disease in men. Lancet 1991;338:464-8. doi:10.1016/0140-6736(91)90542-W.

18 Colditz GA, Manson JE, Hankinson SE. The Nurses' Health Study: 20-year contribution to the understanding of health among women. J Womens Health 1997;6:49-62. doi:10.1089/jwh.1997.6.49.

19 Stunkard AJ, Sørensen T, Schulsinger F. Use of the Danish Adoption Register for the study of obesity and thinness. Res Publ Assoc Res Nerv Ment Dis 1983;60:115-20.

20 Must A, Willett WC, Dietz WH. Remote recall of childhood height, weight, and body build by elderly subjects. Am J Epidemiol 1993;138:56-64.

21 Song M, Hu FB, Spiegelman D, et al. Adulthood weight change and risk of colorectal cancer in the Nurses' Health Study and Health Professionals Follow-up Study. Cancer Prev Res (Phila) 2015;8:620-7. doi:10.1158/1940-6207.CAPR-15-0061.

22 Rich-Edwards JW, Corsano KA, Stampfer MJ. Test of the National Death Index and Equifax Nationwide Death Search. Am J Epidemiol 1994:140:1016-9. 
23 Jones BL, Nagin DS, Roeder K. A SAS procedure based on mixture models for estimating developmental trajectories. Sociol Methods Res 2001:29:374-93doi:10.1177/0049124101029003005.

24 Jones BL, Nagin DS. Advances in group-based trajectory modeling and an SAS procedure for estimating them. Sociol Methods Res 2007:35:542-71 doi:10.1177/0049124106292364.

25 Song M, Willett WC, Hu FB, et al. Trajectory of body shape across the lifespan and cancer risk. Int J Cancer 2016;138:2383-95. doi:10.1002/ ijc.29981.

26 Nagin DS. Group-based modeling of development. Harvard University Press, 2005doi:10.4159/9780674041318.

27 Smith-Warner SA, Spiegelman D, Ritz J, et al. Methods for pooling results of epidemiologic studies: the Pooling Project of Prospective Studies of Diet and Cancer. Am J Epidemiol 2006;163:1053-64. doi:10.1093/aje/kwj127.

28 Stenholm S, Vahtera J, Kawachi I, et al. Patterns of weight gain in middle-aged and older US adults, 1992-2010. Epidemiology 2015;26:165-8. doi:10.1097/EDE.0000000000000228.

29 Engeland A, Bjørge T, Tverdal A, Søgaard AJ. Obesity in adolescence and adulthood and the risk of adult mortality. Epidemiology 2004;15:79-85. doi:10.1097/01.ede.0000100148.40711.59.

30 Must A, Jacques PF, Dallal GE, Bajema C), Dietz WH. Long-term morbidity and mortality of overweight adolescents. A follow-up of the Harvard Growth Study of 1922 to 1935. N Engl J Med 1992;327:1350-5. doi:10.1056/NEJM199211053271904.

31 van Dam RM, Willett WC, Manson JE, Hu FB. The relationship between overweight in adolescence and premature death in women. Ann Intern Med 2006;145:91-7. doi:10.7326/0003-4819-145-2-200607180-00006.

32 Lucas A, Fewtrell MS, Cole TJ. Fetal origins of adult disease-the hypothesis revisited. BM/1999:319:245-9. doi:10.1136/bmi.319.7204.245.

33 Hu FB. Obesity epidemiology.Oxford University Press, 2008doi:10.1093/acprof:0so/9780195312911.001.0001.

34 Keum N, Greenwood DC, Lee DH, et al. Adult weight gain and adiposity-related cancers: a dose-response meta-analysis of prospective observational studies. J Natl Cancer Inst 2015;107:dju428doi:10.1093/jnci/dju428

35 Kodama S, Horikawa C, Fujihara K, et al. Quantitative relationship between body weight gain in adulthood and incident type 2 diabetes: a meta-analysis. Obes Rev 2014;15:202-14. doi:10.1111/obr.12129.

36 Adams KF, Leitzmann MF, Ballard-Barbash R, et al. Body mass and weight change in adults in relation to mortality risk. Am J Epidemiol 2014;179:135-44. doi:10.1093/aje/kwt254.

37 de Mutsert R, Sun Q, Willett WC, Hu FB, van Dam RM. Overweight in early adulthood, adult weight change, and risk of type 2 diabetes, cardiovascular diseases, and certain cancers in men: a cohort study. Am J Epidemiol 2014;179:1353-65. doi:10.1093/aje/kwu052.

38 Goodpaster BH, Delany JP, Otto AD, et al. Effects of diet and physical activity interventions on weight loss and cardiometabolic risk factors in severely obese adults: a randomized trial. JAMA 2010;304:1795802. doi:10.1001/jama.2010.1505.

39 Bouchonville M, Armamento-Villareal R, Shah K, et al. Weight loss, exercise or both and cardiometabolic risk factors in obese older adults: results of a randomized controlled trial. Int) Obes (Lond) 2014;38:423-31. doi:10.1038/ijo.2013.122.
40 Kritchevsky SB, Beavers KM, Miller ME, et al. Intentional weight loss and all-cause mortality: a meta-analysis of randomized clinical trials. PLoS One 2015;10:e0121993. doi:10.1371/journal. pone.0121993.

41 Chiolero A, Faeh D, Paccaud F, Cornuz J. Consequences of smoking for body weight, body fat distribution, and insulin resistance. Am J Clin Nutr 2008;87:801-9.pmid:18400700.

42 Garrison RJ, Feinleib M, Castelli WP, McNamara PM. Cigarette smoking as a confounder of the relationship between relative weight and long-term mortality. The Framingham Heart Study. JAMA 1983;249:2199-203. doi:10.1001/jama.1983.03330400045023.

43 Pischon T, Boeing H, Hoffmann K, et al. General and abdominal adiposity and risk of death in Europe. N Engl J Med 2008;359:210520. doi:10.1056/NEJMoa0801891.

44 Tian J, Venn A, Otahal P, Gall S. The association between quitting smoking and weight gain: a systemic review and meta-analysis of prospective cohort studies. Obes Rev 2015;16:883-901. doi:10.1111/ obr.12304.

45 Williamson DF, Madans J, Anda RF, Kleinman JC, Giovino GA, Byers T. Smoking cessation and severity of weight gain in a national cohort. N Engl J Med 1991;324:739-45. doi:10.1056/ NEJM199103143241106

46 Giovannucci E, Harlan DM, Archer MC, et al. Diabetes and cancer: a consensus report. CA Cancer J Clin 2010;60:207-21. doi:10.3322/ caac.20078.

47 Barone BB, Yeh HC, Snyder CF, et al. Long-term all-cause mortality in cancer patients with preexisting diabetes mellitus: a systematic review and meta-analysis. JAMA 2008;300:2754-64. doi:10.1001/ jama.2008.824.

48 Peairs KS, Barone BB, Snyder CF et al. Diabetes mellitus and breast cancer outcomes: a systematic review and meta-analysis. J Clin Oncol 2011;29:40-6. doi:10.1200/JCO.2009.27.3011.

49 Dehal AN, Newton CC, Jacobs EJ, Patel AV, Gapstur SM, Campbell PT. Impact of diabetes mellitus and insulin use on survival after colorectal cancer diagnosis: the Cancer Prevention Study-II Nutrition Cohort. J Clin Oncol 2012;30:53-9. doi:10.1200/JCO.2011.38.0303.

50 Nagin DS, Odgers CL. Group-based trajectory modeling in clinical research. Annu Rev Clin Psychol 2010;6:109-38. doi:10.1146/annurev. clinpsy.121208.131413.

51 Zheng H, Tumin D, Qian Z. Obesity and mortality risk: new findings from body mass index trajectories. Am J Epidemiol 2013;178:1591-9. doi:10.1093/aje/kwt179.

52 Murayama H, Liang J, Bennett JM, et al. Trajectories of body mass index and their associations with mortality among older japanese: do they differ from those of western populations?Am J Epidemiol 2015;182:597-605. doi:10.1093/aje/kwv107.

53 Hsiao C), Hing E. Use and characteristics of electronic health record systems among office-based physician practices: United States, 2001-2013. NCHS Data Brief 2014;143:1-8.pmid:24439138.

(C) BMJ Publishing Group Ltd 2016

\section{Supplementary materials}

